\title{
Weltstoff und absolute Beharrlichkeit: Die Erste Analogie der Erfahrung und der Entwurf Übergang 1-14 des Opus postumum
}

\author{
von Veit-Justus Rollmann, Frankfurt und Andree Hahmann, Göttingen
}

\begin{abstract}
In Kant's theoretical philosophy substance is not only constitutive for objects as one of the categories of pure reason, but also functions as a substrate for the unity of time. Result of these different functions is an ambiguity in the meaning of Kant's notion of substance. Is there only one or are there many individual substances?

This paper traces the ways in which Kant uses substance not only in the Critique of Pure Reason, but also in the draft „Transition 1-14“ of the Opus postumum to find a solution for this problem.
\end{abstract}

Keywords: Substance, Unity of time, First Analogy of Experience, Opus postumum, Transition 1-14

Heidegger stellt mit Kant heraus, dass die Erfahrung als der alleinige Bereich dessen anzusehen ist, was als gegründete und begründbare Erkenntnis gelten kann. Im System der Grundsätze des reinen Verstandes kann laut Heidegger das „Wesen der Erfahrung“ erblickt werden, insofern dort ein Plan ihres „Wesensbaus“, und damit zugleich die Antwort auf die zentrale Frage nach der Möglichkeit synthetischer Sätze a priori gegeben ist. Heidegger zufolge stellt Kant hier nicht die Frage nach dem Wesen der uns umgebenden Dinge, vielmehr steht hier die Möglichkeit von Erfahrung überhaupt infrage, weshalb das Ding als „Naturding “ zu betrachten ist, d.h. wir haben es in den transzendentalen Grundsätzen lediglich mit den Dingen als Gegenstand einer mathematisch-physikalischen Wissenschaft zu tun. Da die von Kant vorgenommene Bestimmung des Dinges als Naturding in der Art ihres Ansatzes Voraussetzungen einschließt, „die sich auf das Ganze des Seins und den Sinn des Seins überhaupt erstrecken “, ${ }^{1}$ bleibt eine Leerstelle innerhalb der Kantischen Konzeption einer transzendentalen Subjektivität; so ist es dem reinen Verstand nicht möglich, ein einzelnes Ding in der Weise seines alläglichen Begegnens zu bestimmen. Bezogen auf die Dinge als Naturdinge hingegen macht Heidegger deutlich: „Das Ding als Naturding ist nur bestimmbar aus dem Wesen einer Natur überhaupt."2 Für sie gilt, dass das einzelne Ding nur aus einem ihm vorausgehenden Zusammenhang und einer Systematik heraus erkennbar wird. Diese Systematik ist durch das System der Grundsätze ausgesprochen. ${ }^{3}$

1 Heidegger, Martin: Die Frage nach dem Ding. Tübingen 1987, 101.

2 Heidegger (1987), 101.

3 Tuschling weist darauf hin, dass Kant nach eigener Auskunft, d.h. „entsprechend der berühmten Formel, daß die Bedingungen der Möglichkeit der Erfahrung zugleich Bedingungen der Möglichkeit der Gegenstände der Erfahrung sind - eine Gegenstandstheorie, eine 
Für die Konstitution des Dinges von besonderer Bedeutung ist seit Aristoteles die Kategorie der Substanz. Es darf daher nicht verwundern, wenn diese auch bei Kant eine exponierte Stellung einnimmt, und zwar zum einen in ihrer Funktion als reiner Verstandesbegriff und zum anderen im Rahmen der Ersten Analogie der Erfahrung. Mit den unterschiedlichen Funktionen der Substanz innerhalb des kantischen Systems ist das Problem einer gewissen Mehrdeutigkeit des Substanzbegriffs bei Kant verbunden. Handelt es sich bei der Substanz um die eine absolut beharrliche Substanz, worauf die Art und Weise schließen lässt, mit der Kant in der Ersten Analogie mit dem Begriff der Substanz operiert; oder begegnet eine Vielzahl von Substanzen in der Erscheinung, bei welchen die Kategorie der Substanz konstitutiv für Naturdinge im Sinne Heideggers fungiert? Wie verhält es sich schließlich mit einzelnen Erfahrungsgegenständen in der Weise ihres alltäglichen Begegnens? Im Folgenden wird es zunächst darum gehen, die Problematik unterschiedlicher Bedeutungen von Substanz im Ausgang von Kants Erster Analogie der Erfahrung zu exponieren, bevor im Anschluss daran die Übereinstimmungen zwischen der Ersten Analogie und den Entwürfen „Übergang 1-14“ des Nachlasswerkes dargetan werden. Es wird zu zeigen sein, dass Substanz dort nicht in der Auffassung eines konkreten Dings begegnet. Es ist jedoch möglich, den Weltstoff, dessen Existenz Kant in „Übergang 1-14“ mittels einer transzendentalen Argumentation a priori zu beweisen sucht, was seine Funktion anbelangt, mit der einen Substanz, so wie sie in der Ersten Analogie der Erfahrung eingeführt wird, zu identifizieren. Der Weltstoff, den Kant mehrfach als Substanz bezeichnet, ist dort der eine und einzige Gegenstand möglicher Erfahrung, der jedoch selbst, als Gesamtheit des Materials zur Möglichkeit der Erfahrung, kein Gegenstand einer empirischen Anschauung ist. Er ist die eine „Materie überhaupt“, die als Substrat der Erfahrungseinheit, und mithin auch der Zeiteinheit fungiert.

Zum Schluss der Untersuchung soll die Aufmerksamkeit noch einmal auf den von Heidegger herausgestellten Aspekt der kantischen Theorie gelenkt werden, nämlich die Tatsache, dass es Kant nicht um die Dinge in ihrem alltäglichen Begegnen geht, d.h. um die relativ beharrlichen Substanzen, die als einzelne Gegenstände den Horizont unserer alltäglichen Erfahrung ausmachen.

philosophische Theorie der Natur, ihrer Gesetze und des Zusammenhangs der Gegenstände in einer einzigen Natur oder in einer durch den dreidimensionalen Raum und die eindimensionale Zeit konstituierten Welt physischer Gegenstände“ geliefert habe (vgl. hierzu z.B. KU, AA 05: 167.18-168.2). Im Folgenden soll untersucht werden, ob es Kant tatsächlich gelungen ist, eine Gegenstandstheorie zu begründen, und wenn ja, welche Gegenstände von Kant behandelt werden bzw. überhaupt behandelt werden können. Bezüglich des Zusammenhangs der Gegenstände in einer einzigen Natur zumindest, stellt Tuschling heraus, dass ohne den in der KU eingeführten nichtmenschlichen Verstand als einem übersinnlichen Substrat und damit als Grund der absoluten Einheit der transzendentalen Gesetze des reinen Verstandes und der besonderen empirischen Gesetze in einem einzigen System „nicht Eine Natur möglich und konstituiert [wäre], sondern unendlich viele mannigfaltige Naturen“. Tuschling, Burkhard: „System des transzendentalen Idealismus bei Kant? Offene Fragen der - und an die - Kritik der Urteilskraft“. In: Kant-Studien 86, 1995, 197, 206. 


\section{Die Substanz in der Ersten Analogie der Erfabrung}

Zunächst ist festzuhalten, dass Kant den Beweis der Ersten Analogie in der 1781er Ausgabe der Kritik der reinen Vernunft, die in der Folge A-Fassung genannt wird, 1787 in der B-Fassung nicht durch einen anderen Beweisgang ersetzt hat, wie dies im Falle des Deduktions- oder Paralogismuskapitels geschehen ist. ${ }^{4}$ Stattdessen werden diese in die revidierte Fassung übernommen. ${ }^{5}$ Im Folgenden sollen die beiden Fassungen der Grundsätze und die jeweiligen Beweisgänge im Einzelnen behandelt und anschließend miteinander verglichen werden.

1781 schreibt Kant:

Alle Erscheinungen enthalten das Beharrliche (Substanz) als den Gegenstand selbst, und das Wandelbare, als dessen bloße Bestimmung, d.i. die Art, wie der Gegenstand existirt. (KrV, A 182)

Auffallend ist hier die Rede vom „Gegenstand selbst“. ${ }^{6}$ Meint Kant damit das transzendentale Objekt bzw. den transzendentalen Gegenstand? Handelt es sich vielleicht um einen Ding-an-sich-Bezug? Dem Gegenstand selbst bzw. der Substanz wird das Wandelbare gegenübergestellt, das nur als Bestimmung des Gegenstandes existieren kann bzw. lediglich die Art ausdrückt, wie der Gegenstand existiert.

1787 lesen wir dagegen:

Bei allem Wechsel der Erscheinungen beharrt die Substanz, und das Quantum derselben wird in der Natur weder vermehrt noch vermindert. (KrV, B 224)

Auf den ersten Blick scheint die B-Fassung damit etwas ganz anderes als A zu behaupten. Hier wird jeder Vorgang des Entstehens und Vergehens der Substanz ausgeschlossen. Stattdessen ist an dieser Stelle eine Naturkonstante behauptet. ${ }^{7}$ Alle Erscheinungen enthalten nicht länger die Substanz, die auch nicht mehr als der Gegenstand selbst angesprochen wird, stattdessen ist die Substanz das Beharrliche bei jedem Wechsel der Erscheinungen. Bleibt nach dem ersten Teilsatz noch ungeklärt, ob hier eine einzelne, individuelle Substanz gemeint ist, so redet Kant im zweiten Teilsatz von dem Quantum der Substanz. Hier stellt sich zunächst die Frage, was für eine Art Quantum gemeint ist? Handelt es sich um diskrete Einhei-

4 In der Literatur wird häufig behauptet, die B-Fassung gehe weiter bzw. sei stärker als A, was sich schon in der Formulierung des B-Grundsatzes zeigen soll. Vgl. Dicker, Georges: Kant's Theory of Knowledge - An analytical Introduction. Oxford, New York 2004, 145. Diese Vermutung gilt es zu überprüfen.

5 So wurde neben dem Grundsatz lediglich eine kurze einleitende Passage gestrichen, die aber dem Inhalte nach in der vorangestellten B-Fassung enthalten ist.

6 Die Bezeichnung der Substanz als ein Begriff vom Dinge selbst findet sich auch in den Prolegomena: Prol $\$ 25$, AA 04: 307.19-21: „Daher müssen Erscheinungen unter den Begriff der Substanz, welcher aller Bestimmung des Daseins als ein Begriff vom Dinge selbst zum Grunde liegt [...].“

7 Das Verhältnis von Erster Analogie zu den Erhaltungssätzen der Physik behandelt von Weizsäcker, Carl Friedrich: „Kants ,Erste Analogie der Erfahrung und die Erhaltungssätze der Physik“, in: Kant, hrsg. von G. Prauss, Köln 1973, 151-166. Heimsoeth unterstreicht, dass Kant auch schon vor 1781 darum bemüht war, ein Erhaltungsprinzip metaphysisch zu beweisen. Vgl. Heimsoeth, Heinz: Studien zur Philosophie Immanuel Kants I - Metaphysische Ursprünge und ontologische Grundlagen. Bonn 1971, 77-78. 
ten oder ein kontinuierliches Quantum? Und was kann unter einem kontinuierlichen Quantum gedacht werden? Denkbar jedenfalls sind mehrere Alternativen, wie z.B. Materie, Energie (Kraft) oder Bewegung. ${ }^{8}$ Auch von „Natur“, deren Begriff in der KrV den Bezug auf eine Totalität eröffnet, ${ }^{9}$ ist in der A-Fassung des Grundsatzes der Ersten Analogie nicht die Rede.

Offensichtlich liefern die beiden Fassungen des Grundsatzes damit einander widersprechende Konzeptionen der Substanz. Denn wird diese in der Ausgabe aus dem Jahre 1781 mit dem Gegenstand selbst identifiziert, d.h. mit einem bestimmten Etwas, das durch die Kategorie der Substanz bezeichnet werden kann, so bleibt in der Formulierung des Grundsatzes in B offen, was unter der Substanz, deren Quantum in der Natur weder vermehrt noch vermindert werden kann, zu verstehen sei. Man könnte nun denken, dass Kant wohl gut daran getan hat, den A-Grundsatz zu streichen. Nur leider übernimmt Kant die Bestimmung der Substanz als Gegenstand selbst ein wenig später (KrV, A 183/B 227) auch in B. Was für ein konkreter Gegenstand kann hier gemeint sein? Welcher Gegenstand liegt beharrlich jeder Veränderung zugrunde?

Der Zusatz der Substanzerhaltung fehlt in A, wo lediglich behauptet wird, alle „Erscheinungen enthalten das Beharrliche“ und das Wandelbare ist eine bloße Bestimmung der Substanz. Somit erweckt die Formulierung nach A den Anschein, es müsse nur etwas Beharrliches geben, das über einen bestimmten Zeitraum einer Veränderung zugrunde liegt. ${ }^{10}$ Auf den ersten Blick wird dabei nicht die stärkere Behauptung artikuliert, dass sich das Quantum der Substanz ,in der Natur weder vermehrt noch vermindert“. Damit scheint der A-Fassung ein Vorzug gegenüber der B-Fassung zuzukommen, da hier vermeintlich die Widersprüche, die mit der Behauptung der Erhaltung des Quantums der Substanz als Folge einer absoluten Beharrlichkeit der Substanz einhergehen, vermieden werden können.

Ob sich die genannten Probleme tatsächlich mit der A-Fassung umgehen lassen, soll der Vergleich der beiden Beweise miteinander deutlich werden lassen. Denn es fragt sich, ob sich hinter der widersprüchlichen Formulierung der beiden Grundsatzfassungen systemimmanente Schwierigkeiten verbergen, oder ob Kant sich zwischenzeitlich mit anderen Problemen konfrontiert sah, die eine andere Schwerpunktsetzung nötig gemacht haben. Hier soll nur daran erinnert werden, dass die Metaphysischen Anfangsgründe der Naturwissenschaft (MAN), in denen Kant sich in der Behandlung der Mechanik auf die Analogien der Erfahrung stützt, zwischen der ersten und der zweiten Auflage der KrV veröffentlicht worden sind. ${ }^{11}$

8 In der Literatur werden dazu mehrere Lösungen vorgeschlagen, auf die ich später genauer eingehen will.

9 Vgl. hierzu KrV, A 114, sowie B XIX.

10 Thöle sieht hier eine präzisere Formulierung als in B, ,als sie explizit macht, dass das Wandelbare als bloße Bestimmung der beharrlichen Substanz existiert, d.h. dass alles, was nicht beharrlich existiert, nur ein vorübergehender Zustand einer beharrlichen Substanz ist.“ Thöle, Bernhard: „Die Analogien der Erfahrung“. In: Immanuel Kant. Kritik der reinen Vernunft. Hrsg. von Georg Mohr, Marcus Willaschek. Berlin 1998, 275.

11 Saville unterstellt Kant, in der Ersten Analogie zwei verschiedene Ziele zu verfolgen; einmal eine metaphysische Begründung der Newtonschen Physik zu liefern, dann eine vernünftige Rede über einzelne empirische Gegenstände als Substanzen zu begründen. Vgl. 
Heidegger stellt heraus, dass die Beweise der einzelnen Grundsätze, die von Kant in der zweiten Auflage hinzugefügt wurden, einem bestimmten Muster folgen, und zwar weisen diese die Form eines Schlusses auf. Demzufolge lautet der Obersatz: ,Alle Erscheinungen sind in Raum und Zeit'. Der Untersatz hat die Form: ,Nun ist ...' und der Schlusssatz wird eingeleitet mit ,Also ...'

Der Obersatz des B-Beweises der Ersten Analogie lautet nun: „Alle Erscheinungen sind in der Zeit, in welcher, als ihrem Substrat, (als beharrlicher Form der inneren Anschauung,) das Zugleichsein sowohl als die Folge allein vorgestellt werden kann. Die Zeit also, in der aller Wechsel der Erscheinungen gedacht werden soll, bleibt und wechselt nicht; weil sie dasjenige ist, in welchem das Nacheinander- oder Zugleichsein nur als Bestimmung derselben vorgestellt werden können. "12 Die beharrliche Form aller Erscheinungen, insofern sie wahrgenommen werden, ist die Zeit als Form des inneren Sinnes, d.h. jede mögliche Anschauung ist in der Zeit. Wir können Erscheinungen nur deshalb in eine zeitliche Ordnung bringen, weil die Zeit selbst konstant ist. Sie ist nicht wandelbar, d.h. sie bleibt und wechselt nicht.

Der Untersatz lautet: „Nun kann die Zeit für sich nicht wahrgenommen werden."13 Die Feststellung der Nichtwahrnehmbarkeit der Zeit ist ein wichtiger Punkt in der kantischen Argumentation. Es handelt es sich dabei um eine Schlüsselstelle in der Argumentation, auf die sich die anschließenden Folgerungen stützen. Heidegger erkennt in der kantischen Feststellung, dass die Zeit an sich nicht wahrgenommen werden kann, die „eigentliche Angel, in der sich der Beweis dreht". ${ }^{14}$

Als Schlusssatz ist zu lesen:

Folglich muß in den Gegenständen der Wahrnehmung, d.i. den Erscheinungen, das Substrat anzutreffen sein, welches die Zeit überhaupt vorstellt, und an dem aller Wechsel oder Zugleichsein durch das Verhältniß der Erscheinungen zu demselben in der Apprehension wahrgenommen werden kann. Es ist aber das Substrat alles Realen, d.i. zur Existenz der Dinge Gehörigen, die Substanz, an welcher alles, was zum Dasein gehört, nur als Bestimmung kann gedacht werden. Folglich ist das Beharrliche, womit in Verhältniß alle Zeitverhältnisse der Erscheinungen allein bestimmt werden können, die Substanz in der Erscheinung, d.i. das Reale derselben, was als Substrat alles Wechsels immer dasselbe bleibt. ${ }^{15}$

Saville, Anthony: Kant's Critique of pure Reason - an Orientation to the central Theme. Malden, Oxford, Victoria 2005, 70; siehe auch Kim, Jaeho: Substanz und Subjekt - Eine Untersuchung der Substanzkategorie in Kants „Kritik der reinen Vernunft“. Würzburg 2006, 114. Die Nähe zwischen den Analogien der Erfahrung und den MAN wird von vielen Interpreten hervorgehoben, so auch Westphal, Kenneth R.: Kant's Transcendental Proof of Realism. Cambridge 2004; Allison, Henry E.: Kant's Transcendental Idealism. New Haven, London 2004. Longuenesse will gar eine Konfusion der Ersten Analogie mit den MAN erkennen und behauptet, dass es Kant gar nicht möglich sei, in der KrV einen Substanzerhaltungssatz aufzustellen. Vgl. Longuenesse, Béatrice: Kant and the Capacity to Judge: Sensibility and Discursivity in the Transcendental Analytic of the Critique of Pure Reason. Princeton 1998, 345-346.

$12 \mathrm{KrV}, \mathrm{B}$ 224-225.

$13 \mathrm{KrV}, \mathrm{B} 225$.

14 Heidegger (1987), 181.

$15 \mathrm{KrV}, \mathrm{B} 225$. 
Das Substrat muss in den Gegenständen der Wahrnehmungen, d.h. den Erscheinungen, angetroffen werden. Nur an dem Substrat sind der Wechsel und das Zugleichsein der Erscheinungen durch ihr Verhältnis zu demselben in der Apprehension vorstellbar, d.h. Folge und Zugleichsein können nur in dem Verhältnis zu diesem Substrat wahrgenommen werden. Mit diesem Substrat, als dem beharrlichen Substrat jeder Veränderung, ist die Kategorie der Substanz, und zwar in ihrer schematisierten Form ausgesagt. ${ }^{16}$ Also ist in den Erscheinungen die Substanz anzutreffen, die absolut beharrlich ist und die jeder möglichen Veränderung als Substrat zugrunde liegt. Aus der absoluten Beharrlichkeit und damit der Identität zu jeder Zeit folgt zugleich die Erhaltung ihres Quantums. Die Substanzerhaltung muss folglich als Konsequenz der absoluten Beharrlichkeit erachtet werden, die wiederum notwendig ist, um die Vorstellbarkeit der Einen Zeit gewährleisten zu können.

Es fragt sich nun, ob diese Schwierigkeiten in der ersten Auflage vermieden werden können, immerhin redet Kant hier von der Substanz als dem Gegenstand selbst und behauptet nicht die Erhaltung eines Quantums der Substanz in der Natur. Bei der Betrachtung des A-Beweises fällt zuerst auf, dass dieser zumindest auf den ersten Blick sehr viel komplexer als der B-Beweis daherkommt, wohingegen

16 Genau hier liegt ein ernsthaftes Problem in der kantischen Philosophie, das Heidegger in seinem ersten Buch zu Kant schließlich zu der Folgerung verleitet hat, die Vernunft sei im Schematismus selbst gegründet und müsse daher ihres Führungsanspruches enthoben werden. Denn wie kann ein reiner Verstandesbegriff auf die reine Anschauung übertragen werden? Handelt es sich hier nicht um zwei gänzlich verschiedene Stücke der Erkenntnis: Anschauung und Denken? (vgl. Heidegger 1987, 174). Im Schematismus-Kapitel lesen wir, dass die reinen Verstandesbegriffe „in Vergleichung mit empirischen (ja überhaupt sinnlichen) Anschauungen, ganz ungleichartig“ (KrV, A 137/B 176) sind. Stattdessen muss es ein Drittes geben, „was einerseits mit der Kategorie, andererseits mit der Erscheinung in Gleichartigkeit stehen muss, und die Anwendung der ersteren auf die letztere möglich macht" (KrV, A 138/B 177). Empirische Begriffe enthalten im Gegensatz zu den Kategorien in sich bereits ein Schema ihrer Anwendung. (KrV, A 141/B 180). Reine Begriffe aber bedürfen ihres Schemas, um auf eine Erscheinung appliziert werden zu können. Dieses Vermittelnde ist eine Vorstellung, die zwar rein, aber gleichermaßen intellektuell wie auch sinnlich sein muss. Die Charakterisierung der Zeit als beharrliches Substrat, das selbst bleibt und nicht wechselt und an dem allein Zugleichsein und Folge möglich sein sollen, ermöglicht es Kant, die Substanzkategorie unter ihren schematisierten Bedingungen anzuwenden. Wieso das? Kant hat in der transzendentalen Doktrin der Urteilskraft zwei Hauptstücke unterschieden, einmal die Behandlung der sinnlichen Bedingungen, unter welchen allein die reinen Verstandesbegriffe appliziert werden können - das ist der Schematismus der reinen Verstandesbegriffe - und dann die Frage nach den synthetischen Urteilen, „welche aus reinen Verstandesbedingungen unter diesen Bedingungen [Hervorhebung: Vf.] a priori herfließen“ KrV, A 136/B 175. Demzufolge sind beide Teile der Behandlung untrennbar miteinander verbunden. Die Analogien der Erfahrung gehören zu den Grundsätzen des reinen Verstandes und somit zu dem zweiten von Kant herausgestellten Bereich. Demgemäß müsste auch in der Ersten Analogie zweierlei anzutreffen sein: Erstens das Schema der Substanz als sinnliche Restriktion der Kategorie, d.h. die sinnliche Bedingung, unter der allein die Kategorie der Substanz angewendet werden kann, zweitens die Kategorie selbst. In der Formulierung des Grundsatzes nun will sich Kant der Kategorie bedienen, in der Ausführung, d.h. der Anwendung auf die Erscheinung, soll hingegen das Substanzschema „als [...] Schlüssel ihres Gebrauchs“ Anwendung finden. 
der B-Beweis klarer und stringenter aufgebaut ist. ${ }^{17}$ Doch damit ist noch nicht gesagt, dass Kant tatsächlich anderes behauptet. ${ }^{18}$

Der A-Beweis beginnt genauso wie der B-Beweis mit der Feststellung, dass alle Erscheinungen in der Zeit sind, in der allein Nacheinander- und Zugleichsein vorgestellt werden können (vgl. KrV, A 182, dieser Satz ist in B getilgt worden). In B folgt die für die Argumentation wichtige Feststellung der Nichtwahrnehmbarkeit der Zeit. A führt stattdessen die Sukzessivität der Apprehension ein, aus der keine Notwendigkeit im Zeitverlauf geschlossen werden kann (vgl. KrV, A 182/B 225). Im B-Beweis des Allgemeinen Grundsatzes der Analogien der Erfahrung werden beide Bedingungen angeführt, d.h. sowohl die Nichtwahrnehmbarkeit der Zeit als auch die Unmöglichkeit, allein aufgrund der Apprehension eine objektive Zeitbestimmung vorzunehmen (vgl. KrV, B 219). ${ }^{19}$ Bis zu diesem Punkt kann die kantische Argumentation in A wie in B als unproblematisch gelten, sofern man die Grundannahmen der kantischen Theorie akzeptiert, nämlich davon ausgeht, dass Raum und Zeit die beharrlichen Formen der Anschauung sind, wobei der Raum allen äußeren und die Zeit allen inneren wie äußeren Anschauungen zugrunde liegt und die Kantische These, der zufolge Wahrnehmung ohne Verstandesbegriffe für eine objektive Erkenntnis (Erfahrungsurteile) unzureichend ist, als unstrittig betrachtet. ${ }^{20}$ In B schließt Kant an die Feststellung, dass die Zeit für sich nicht wahrgenommen werden kann, die These an, es müsse in den Gegenständen der Wahrnehmung das Substrat anzutreffen sein, welches die Zeit überhaupt vorstellt. Aller Wechsel - so die These Kants - könne nur im Verhältnis zu diesem Substrat vorgestellt werden. Mit dem so charakterisierten Substrat wird schließlich die Substanz identifiziert, die als das Reale der Erscheinungen immer dieselbe bleibt

17 Dass der B-Beweis klarer ist, behauptet auch Longuenesse und ebenso, dass es keine wesentlichen Unterschiede zwischen beiden Beweisen gibt (vgl. Longuenesse 1998, 335). Jedoch ist ihrer Meinung nach die Behauptung eines Erhaltungssatzes eine Konfusion der $\mathrm{KrV}$ mit den MAN, da es Kant in der KrV nicht möglich sein soll, eine quantitative Substanzerhaltung zu behaupten. Dabei scheint sie nicht zu berücksichtigen, dass die quantitative Erhaltung aus der qualitativen Identität der Substanz folgt. Vgl. Longuenesse (1998), 345-346.

18 In der Rekonstruktion des A-Beweises soll nun ein besonderes Augenmerk darauf gerichtet sein, ob Kant auch hier eine absolute Beharrlichkeit behauptet oder ob der A-Beweis ohne diese auskommt und eine relativ beharrliche Substanz ausreicht, ein schlüssiges Argument zu liefern.

19 Für Guyer sind die Feststellung der Nichtwahrnehmbarkeit der Zeit und der Hinweis auf die Sukzessivität der Apprehension äquivalente Argumentationsschritte, da durch die Behauptung in A gesagt wird, dass in der sukzessiven Folge der Apprehension nichts anzutreffen ist, was die Beharrlichkeit der Zeit ausdrücken kann. Vgl. Guyer, Paul: Kant and the Claims of Knowledge. Cambridge, New York u.a. 1987, 217. Diese Interpretation setzt jedoch voraus, dass Kant die Zeit mit der Beharrlichkeit identifiziert, wie Guyer selbst kurz zuvor behauptet - Kant sagt aber, dass das Beharrliche notwendig ist, um zu bestimmen, „ob diese Mannigfaltige, als Gegenstand der Erfahrung, zugleich sei, oder nach einander folge“ (KrV, A 182/B 225).

20 Selbst Hume würde Kant zumindest den zweiten Punkt dieser Voraussetzungen zugestehen, hat er doch gerade darauf hingewiesen, dass Erfahrung uns keine notwendigen Schlüsse belegen kann. 
und deren Quantum in der Natur weder vermehrt noch vermindert werden könne. In A behauptet Kant nun entsprechend, dass etwas zugrunde liegen müsse, „was jederzeit ist, d.i. etwas Bleibendes und Beharrliches, von welchem aller Wechsel und Zugleichsein nichts, als so viel Arten [...] sind, wie das Beharrliche existirt" (KrV, A 182). Dieses Beharrliche erklärt Kant zum „Substratum der empirischen Vorstellung der Zeit selbst, an welchem alle Zeitbestimmung allein möglich ist. Die Beharrlichkeit drückt überhaupt die Zeit als das beständige Correlatum alles Daseins der Erscheinungen, alles Wechsels und aller Begleitung, aus." (KrV, A 183/B 226) Nur unter Zugrundelegung dieses Beharrlichen können Zeitverhältnisse überhaupt möglich sein. Hier ist also die Rede von einem beharrlichen Substrat, das jederzeit ist, von dem Folge und Zugleichsein zwei Existenzweisen sind, das der alleinige Grund der Zeitbestimmung und damit das Substrat der empirischen Vorstellung der Zeit überhaupt ist. „Durch das Beharrliche allein bekommt das Dasein in verschiedenen Theilen der Zeitreihe nacheinander eine Größe, die man Dauer nennt. Denn in der bloßen Folge allein ist das Dasein immer verschwindend und anhebend und hat niemals die mindeste Größe. Ohne dieses Beharrliche ist also kein Zeitverhältniß." (KrV, A 183/B 226) Nur in Bezug auf ein immer da seiendes Substrat, und mithin auf einen absoluten Parameter, ist der Wechsel der Erscheinungen bestimmbar, und nur so kann eine relative Dauer ausgemacht werden. Damit dürfte klar sein, dass auch im A-Beweis nicht von einer relativen Beharrlichkeit die Rede ist, sondern genauso wie in $\mathrm{B}$ die absolute Beharrlichkeit der Substanz als Voraussetzung jeder relativen Beharrlichkeit, die nur im Bezug zu einem absoluten Parameter als relative Dauer bestimmt werden kann. ${ }^{21}$ Nun folgert Kant sogar:

[...] mithin ist dieses Beharrliche an den Erscheinungen das Substratum aller Zeitbestimmung, folglich auch die Bedingung der Möglichkeit aller synthetischen Einheit der Wahrnehmungen, d.i. der Erfahrung, und an diesem Beharrlichen kann alles Dasein und aller Wechsel in der Zeit nur als ein modus der Existenz dessen, was bleibt und beharrt, angesehen werden. Also ist in allen Erscheinungen das Beharrliche der Gegenstand selbst, d.i. die Substanz (phaenomenon); alles aber, was wechselt oder wechseln kann, gehört nur zu der Art, wie diese Substanz oder Substanzen existiren, mithin zu ihren Bestimmungen. (KrV, A 183-184/B 226-227)

Die letzten beiden Sätze sind konsequente Folgerungen Kants aus dem bisher ausgeführten Beweis. Denn ohne das Beharrliche ist nach Kant kein Zeitverhältnis und somit letztlich keine synthetische Einheit der Wahrnehmungen, d.h. keine Erfahrung möglich. Damit glaubt Kant sein Beweisziel erreicht zu haben, was sich im Wiederaufgreifen des Grundsatzes ausdrückt. Auffallend ist hier der schwankende Sprachgebrauch Kants. So spricht er an dieser Stelle von der Substanz, den Substanzen und dem Gegenstand selbst als Substanz, wohingegen in der Formulierung des Grundsatzes lediglich von der Substanz bzw. dem Gegenstand selbst die Rede war.

21 Vgl. z.B. Strawson, Peter Frederick: The Bounds of Sense - An Essay on Kant's Critique of Pure Reason. London 1966, 129. 


\section{Eine Zeit, Eine Erfahrung und Eine Substanz}

Der schwankende Sprachgebrauch gibt einen Hinweis darauf, dass Kant zwei verschiedene Substanzbegriffe verwendet, und zwar einen Individualbegriff und den Begriff der einen Substanz, der sich auf das Ganze der Natur bezieht. Diese eine Substanz ist auch in der kantischen Behauptung angesprochen, dass sich das Quantum der Substanz zu aller Zeit erhalte. Ist es aus der Perspektive des kantischen Systems überhaupt zulässig, eine solche These aufzustellen?22 Und was kann sich durchgängig erhalten, sowohl qualitativ als auch quantitativ, etwa Materie? Kant selbst legt den Schluss auf die Materie nahe und in den Beispielen, die er referiert, wird dieser auch explizit ausgesprochen. Doch kann diese Materie überhaupt noch ein möglicher Erfahrungsgegenstand sein oder nicht, bzw. kann es sich damit noch um einzelne, konkrete Substanzen handeln? Kant stößt somit auf eine der Hauptschwierigkeiten der Ersten Analogie: Ist die Substanz eine oder viele? Dieses Problem ist der Sekundärliteratur nicht verborgen geblieben. ${ }^{23}$ Scheinbar übersteigt die Substanz, so wie diese von Kant in der Ersten Analogie vorgestellt wird, jeden einzelnen empirischen Gegenstand, ja sogar jede konkrete Gegenständlichkeit überhaupt. Schon in der Formulierung des Grundsatzes von 1781 genauso wie 1787 wird von der Substanz gesprochen. Die Frage, ob es sich um eine oder mehrere Substanzen handelt, ist eng mit einer zweiten Frage verbunden, nämlich ob es sich bei der Substanz um Materie handelt und wenn ja, was für eine Materie. ${ }^{24}$ „Denn von den einzelnen Dingen der Alltagswelt steht unbezweifelbar

22 Longuenesse z.B. hat dies betreffend erhebliche Bedenken. Vgl. Longuenesse (1998), 345-346.

23 So behauptet z.B. Sans: „Die erste Analogie liefert jedenfalls keinen Grund, einzelne Dinge als verschiedene Substanzen aufzufassen. In der Welt der Erfahrung gibt es keine Individuen. Die objektive Wirklichkeit bestünde aus der Menge aller Bestimmungen, die augenscheinlich als Attribute einer einzigen Substanz verstanden werden müssen." Sans, Georg: Ist Kant's Ontologie naturalistisch? Die Analogien der Erfahrung in der Kritik der reinen Vernunft. Stuttgart, Berlin, Köln 2000, 54, vgl. auch Sans, Georg: „Wie viel Substanz braucht Kant? “, in: Revista Portuguesa de Filosofia 61, 2005; ähnliche Bedenken finden sich bei Westphal (2005), 161-164; Tuschling, Burkhard: „Widersprüche im transzendentalen Idealismus“, in derselbe: Probleme der „Kritik der reinen Vernunft“ - KantTagung Marburg 1981. Berlin, New York 1984, 227-311; Dicker (2004), 149; Strawson, Peter Frederick: „Kant on Substance“, in derselbe: Entity and Identity. Oxford 1997, 269-270. Für von Weizsäcker ist die Behauptung der einen Substanz eigentlich kein Problem, sondern rückt die Erste Analogie in die Nähe der Erhaltungssätze der Physik. Vgl. von Weizsäcker (1973), 157-158. Siehe auch Rosenberg, Jay F.: Accessing Kant - A Relaxed Introduction to the Critique of Pure Reason. New York 2005 und Cramer, Konrad: Nicht-reine synthetische Urteile a priori. Ein Problem der Transzendentalphilosophie Immanuel Kants. Heidelberg 1985.

24 Allison zumindest lehnt die Deutung der von Kant hier angesprochenen Materie als Aristotelische Hyle strikt ab, stattdessen schlägt er vor, die kantische Aussage zur Substanzerhaltung so zu deuten, dass lediglich die Kategorie der Quantität auf eine - von Kant an anderer Stelle durch die Eigenschaft der Ausdehnung charakterisierte - Materie angewendet werden kann. Vgl. Allison (2004), 245. Gegen diese Deutung muss eingewendet werden, dass nicht bloß eine einzelne Kategorie angewendet werden kann, um einen Gegenstand zu bestimmen. Dieser muss dagegen durch alle Titel bestimmt sein. Vgl. Refl 5932, AA 18: 392.03-09: „Also ist Categorie der Begrif von einem obiecte überhaupt, so fern es in An- 
fest, daß sie anfangen und aufhören zu existieren. Demzufolge wären konkrete Dinge - Tische und Stühle, Tiere und Menschen - keine Substanzen, sondern Akzidenzien. Am Ende bleibt als einziger Kandidat einer Substanz (phaenomenon) die Materie übrig, aus der alle Gegenstände gebildet sind. Allein Materie kann sich verändern, ohne selbst zu entstehen oder zu vergehen. " 25 Jedenfalls scheint es zumindest in der Ersten Analogie für „[...] die Annahme von mehr als einer einzigen Substanz (phaenomenon) [...] keinen Grund zu geben." 26 Es kann sich bei dem absolut beharrlichen Substrat der Zeitvorstellung um keinen individuellen Gegenstand mehr handeln. Es fragt sich daher, ob die Substanz in der Ersten Analogie nicht eine einzige sein muss.

Wir können festhalten, dass wir es hier mit mindestens zwei einander widersprechenden Substanzbegriffen zu tun haben: einmal die Substanz als den Gegenstand selbst und zum anderen die Substanz als das absolut beharrliche Substratum, das mit sich selbst identisch jeder möglichen Veränderung zugrunde liegt, dessen Quantum daher weder vermehrt noch vermindert werden kann und nur auf diese Weise die Zeit überhaupt vorstellbar macht. Ist ein „Gegenstand überhaupt" mit dem Dasein zu aller Zeit vereinbar? Wie können das immerwährende Dasein, das Substrat aller Zeitbestimmung, das beharrlich jedem Wechsel zugrunde liegt, und die Substanz als Gegenstand selbst, die konkrete, bestimmte Erscheinung, miteinander vereinbar sein? Kurz gesagt, wie kann ein immerwährendes Dasein zugleich ein bestimmter Gegenstand sein?

Im Folgenden soll gezeigt werden, dass die in der Literatur erhobenen Zweifel durchaus berechtigt sind und ihren Grund in einer widersprüchlichen Substanzkonzeption haben, danach kann die Substanz aufgrund der inhaltlichen bzw. logischen Bestimmung sowie ihrer Funktion in der Ersten Analogie und schließlich ihrer ontologischen Konstitution nur eine einzige sein. Zugleich jedoch ist es not-

sehung einer logischen Function der Urtheile a priori an sich bestimmt ist (daß man [es durch keine andere] durch diese Function die Verbindung des Manigfaltigen in seiner Vorstellung denken muß). Alle obiecte, die wir denken sollen, müssen in Ansehung aller logischen Functionen des Verstandes bestimmt seyn. "Sans hingegen versteht den Substanzerhaltungssatz im Lichte eines numerischen Identitätssatzes, wo zwar die Identität behauptet wird, allerdings nicht einer Vielzahl von numerisch unterschiedenen Substanzen. Vgl. Sans (2000), 58. Eine quantitative Erhaltung behauptet Thöle, der sich dazu auf die MAN stützt. Thöle (1998), 275-276. Thöle scheint hier zu übersehen, dass man sich nicht auf die MAN stützen darf, um ein Problem der KrV erklären zu wollen. Dabei wird vergessen, dass die MAN die $\mathrm{KrV}$ voraussetzt, es würde sich also um eine petitio principii handeln. Hinzu kommt, dass die Substanz, die Kant in den MAN mit der Materie identifiziert, durch das empirische Prädikat der Bewegung individuiert wird. Die Substanz in der $\mathrm{KrV}$ hingegen transzendental bestimmt sein muss, daher nicht identisch mit der materiellen Substanz der MAN ist.

25 Sans (2000), 59. Für Sans ist die kantische Position materialistisch: „Die Rede von dem gleichbleibenden Quantum der Materie und die Berichtigung des Begriffs der Veränderung sind starke Indizien, daß Kant sich insgeheim auf eine monistische und materialistische Deutung der ersten Analogie festgelegt hat." Sans (2000), 59. Was bedeutet in diesem Zusammenhang materialistisch? Kant spricht nur von der Materie in der Erscheinung, d.h. so wie sie in Raum und Zeit als den beiden idealen, subjektiven Anschauungsformen bestimmt ist.

26 Sans (2000), 60. 
wendig, eine Vielheit der Substanzen anzunehmen, um die Erfahrung der konkreten Gegenständlichkeit zu konstituieren.

Bezüglich der Funktion der Substanz in der Ersten Analogie wurde herausgestellt, dass sie die eine Zeit vorstellbar machen soll, andernfalls ist nach Kant nicht die eine Erfahrung als ein nach Gesetzen geordnetes, notwendiges Ganzes möglich. ${ }^{27}$ In dieser Funktion ist die Substanz als eine notwendige Bedingung der Möglichkeit der Erfahrung anzusehen (KrV, A 183/B 226-227). Ohne die Substanz, „fiele dasjenige weg, welches die Einheit der Zeit allein vorstellen kann, nämlich die Identität des Substratum, als woran aller Wechsel allein durchgängige Einheit hat" (KrV, A 186/B 229). ${ }^{28}$ Damit korrespondiert die Substanz in gewisser Hinsicht der transzendentalen Einheit der Apperzeption als dem Grund der Einheit des Bewusstseins in ihrer Funktion innerhalb des Kantischen Systems der Bedingungen möglicher Erfahrung. Denn genauso wie die transzendentale Einheit der Apperzeption die Einheit des Bewussteins in jeder möglichen Erfahrung distributiv bestimmt, determiniert die Substanz die Identität des Substratums jeder möglichen Zeitvorstellung sowohl distributiv als auch kollektiv, und damit jeder möglichen Erfahrung überhaupt, insofern Erfahrung notwendig zeitlich bestimmt ist.

Es stellt sich nun die Frage, inwiefern die eine Zeit durch eine Vielzahl von Substanzen zum einen vorstellbar gemacht und darüber hinaus in ihrer Einheit er-

$27 \mathrm{Vgl} . \mathrm{KrV}$, A 110: „Es ist nur eine Erfahrung, in welcher alle Wahrnehmungen als im durchgängigen und gesetzmäßigen Zusammenhange vorgestellt werden: eben so wie nur ein Raum und Zeit ist, in welcher alle Formen der Erscheinung und alles Verhältniß des Seins oder Nichtseins statt finden. Wenn man von verschiedenen Erfahrungen spricht, so sind es nur so viel Wahrnehmungen, so fern solche zu einer und derselben allgemeinen Erfahrung gehören.“; KrV, B 218: „Erfahrung ist nur durch die Vorstellung einer nothwendigen Verknüpfung der Wahrnehmungen möglich.“ Vgl. KrV, A 188-189/B 232: „Denn es ist nur Eine Zeit, in welcher alle verschiedene Zeiten nicht zugleich, sondern nach einander gesetzt werden müssen.“ Ferner KrV, A 169/B 211: „Raum und Zeit sind quanta continua, weil kein Theil derselben gegeben werden kann, ohne ihn zwischen Grenzen (Punkten und Augenblicken) einzuschließen, mithin nur so, daß dieser Theil selbst wiederum ein Raum oder eine Zeit ist. Der Raum besteht also nur aus Räumen, die Zeit aus Zeiten. Punkte und Augenblicke sind nur Grenzen, d.i. bloße Stellen ihrer Einschränkung [...]." Vgl. eine selbstständige Reflexion im Handexemplar der KrV: Refl LXXIX E 32 - A 182, AA 23: 30.11-17: „Der Wechsel kan nur durchs Beharrliche und als dessen Veränderung wargenommen werden. Denn der Unterschied der Zeiten, darin Dinge seyn, kan nur in ihnen als Theilen einer und derselben Zeit wargenommen werden. Aller Wechsel ist nur Zeiteintheilung. Daher muß etwas seyn, was die ganze Zeit hindurch ist, weil das Ganze der Theilung immer zum Grunde liegt. Daher ist Substanz das Substrat, und das Wechselnde ist nur die Art desselben zu existiren. "Im OP heißt es dann [OP, AA 22: 91.07-08]: „Es ist nur Ein Raum u. Eine Zeit (darin besteht die Unendlichkeit) und nur Eine Erfahrung “; OP, AA 22: 426.21-22: „Es ist nur Ein Raum und nur Eine Zeit und Eine Materie welche jenen erfüllt [...].“; OP, AA 21: 224.03-04: „Es ist nur Ein Raum und nur Eine Zeit und nur Eine Materie in welcher alle Bewegung angetroffen wird."

28 Für Dicker ist es möglich, dass die eine Substanz die Identität der Zeit sicherstellen und diese zugleich vorstellbar machen kann: „Strictly speaking, it would seem that for time to have a perceptual stand-in, by reference to which relations of simultaneity and succession can be determined, only one substance is needed, so the ,single stuff' interpretation would fit Kant's purpose.“ Vgl. Dicker (2004), 149. Hier soll jedoch gezeigt werden, dass es nicht nur möglich, sondern notwendig ist, die eine Substanz anstelle der vielen anzunehmen. 
zeugt werden kann. Die Zeit an sich ist nicht wahrnehmbar, wie Kant entschieden erklärt. Wie kann nun eine Vielheit von Substanzen die „Eine Zeit“ vorstellen, wenn nicht wiederum eine ihnen zugrunde liegende Zeit angenommen wird, die den Ablauf der Zeit an den einzelnen Substanzen synchronisiert? Wird diese allerdings vorausgesetzt, erneuert sich auch das Problem, dass die Zeit nur durch eine Substanz vorstellbar gemacht werden kann, weil auch die neuerdings zugrunde liegende Zeit an sich selbst leer ist und nicht wahrgenommen werden kann. Die Annahme einer Vielheit von Substanzen führt auf diese Weise zu einem Regress, der nur durch eine an sich selbst wahrnehmbare Zeit vermieden werden kann, d.h. eine Zeit, die selbst ein Gegenstand der Erfahrung ist, oder die eine Substanz, die die Funktion der Einheit in der Zeitvorstellung übernimmt. Dem möglichen Einwand, dass dieser Regress auch durch ein Prinzip der Synchronisation zwischen den einzelnen Substanzen vermieden werden könne, wie z.B. durch das Kausalitätsprinzip oder das Prinzip der Wechselwirkung, muss entgegnet werden, dass beide Prinzipien die Erste Analogie allererst voraussetzen. Denn in der Ersten Analogie wird die Einheit des Substrats herausgestellt, ${ }^{29}$ an dem die Zeit vorstellbar und die Einheit der Erfahrung damit allein möglich ist. Gibt es diese eine Substanz nicht, kann auch die eine Zeit nicht vorgestellt werden, die den Ablauf der Zeiten in bzw. an den einzelnen Substanzen garantiert und das Verhältnis der Substanzen zueinander synchronisiert. Gerade das muss aber angenommen werden, wenn ich den Ablauf der Zeit durch eine Vielheit von Substanzen vorstellbar mache. Wer würde sonst versichern können, dass die zeitliche Bestimmung in der Synthesis der Anschauung nicht nur an einem Teil der Substanzen oder vielleicht auch nur einer Substanz vollzogen wird, die nicht die gleiche Zeit mit den anderen Substanzen teilt? ${ }^{30}$ Es wäre sogar möglich, dass selbst hier auf der Erde Substanzen in verschiedenen Regionen und Orten unterschiedliche Zeiten vorstellen, so

29 Kant selbst spricht von der Identität des Substratums, das notwendig zu jeder möglichen Zeitbestimmung vorausgesetzt werden muss. Vgl. KrV, A 186/B 229: „Denn alsdenn fiele dasjenige weg, welches die Einheit der Zeit allein vorstellen kann, nämlich die Identität des Substratum, als woran aller Wechsel allein durchgängige Einheit hat.“

30 Es ist z.B. denkbar, dass eine Vielzahl von Substanzen auf der Erde eine Zeit repräsentiert, die in ihrem Ablauf von der Zeit abweicht, die von einer Anzahl von Substanzen auf einem anderen Planeten vorstellbar gemacht wird, sodass ein Mensch, der die Erde verlässt und dorthin kommt, eine andere Zeit als auf der Erde erfährt. Unsere Erfahrung würde sich danach fundamental von der seinigen unterscheiden. Aus heutiger Sicht stellt sich natürlich die Frage, ob die allgemeine Relativitätstheorie, die genau das behauptet, die Kantische Konzeption der Einheit der Erfahrung aufhebt. Dem ließe sich allerdings erwidern: Setzt nicht die Behauptung, dass es unterschiedliche Zeiten an unterschiedenen RaumZeitstellen im Universum gibt, die Eine Zeit voraus, woran die Unterschiede überhaupt erst sichtbar gemacht werden können? In ähnlicher Weise argumentiert Beck für den Satz der Kausalität in Beziehung auf die Unbestimmtheitstheorie aus der Quantenmechanik. Denn um die Unbestimmtheit von Quantenteilchen bestimmen zu können, muss der Satz der Kausalität und somit die Zweite Analogie seiner Meinung nach vorausgesetzt werden. „Aus diesem Grunde halte ich dafür, daß es gute epistemologische Gründe dafür gibt, unser Wissen um die Unbestimmtheit als einen Parasiten unseres Wissens um die kausale Bestimmtheit zu betrachten.“ Beck, Lewis White: „Die Zweite Analogie und das Prinzip der Unbestimmtheit“, in: Kant - Zur Deutung seiner Theorie von Erkennen und Handeln, hrsg. von Gerold Prauss, Köln 1973, 172. Siehe auch Anm. 27. 
dass praktisch jeder seine eigene Erfahrung hätte. ${ }^{31}$ Das widerspräche aber den kantischen Voraussetzungen, wonach es nur eine Erfahrung geben kann, ${ }^{32}$ deren objektive Gültigkeit aus den subjektiven Bedingungen der Möglichkeit einer Erfahrung gewonnen wird. ${ }^{33}$ Die eine Erfahrung erfordert also die eine Substanz als Substrat aller Zeitbestimmung, genauso wie die eine Zeit, als deren Substrat die eine Substanz dienen soll.

Als das empirische Kriterium der Substanz stellt Kant in der Zweiten Analogie die Kausalität vor (vgl. KrV, A 204/B 249). Denn „wo Handlung mithin Thätigkeit und Kraft ist, da ist auch Substanz, und in dieser allein muß der Sitz jener fruchtbaren Quelle der Erscheinungen gesucht werden“ (KrV, A 204/B 250). Für Kant besteht eine enge Verbindung zwischen den Begriffen Kausalität, Handlung, Kraft und Substanz (vgl. KrV, A 204/B 249). Die enge Verbindung, die Kant zwischen Substanz und Kraft bzw. Kausalität sieht, hat er von Leibniz geerbt. ${ }^{34}$

Indem Kant an dieser Stelle die Substanz zum ersten bzw. letzten Subjekt der Handlung erklärt, werden von ihm zwei unterschiedliche Ebenen miteinander in Verbindung gebracht. So ist Substanz einmal ein „Etwas, das als Subject, niemals aber als bloßes Prädicat existiren könne, wovon ich gar nicht weiß, ob es irgend ein Ding geben könne, das dieser Gedankenbestimmung correspondirte, wenn nicht empirische Anschauung mir den Fall der Anwendung gäbe“ (KrV, B 149). ${ }^{35}$ Damit ist die Kategorie der Substanz in ihrer logischen Funktion angesprochen, d.h. in einem gegebenen Urteil eine eindeutige Bestimmung der Subjektstelle zu liefern. Andererseits offenbart sich für Kant die Substanz in der Anschauung am einfachsten durch Handlung, denn sie ist das letzte oder erste Subjekt der Kausalität. ${ }^{36}$ Als

31 Dass tatsächlich die Zeit an verschiedenen Orten unterschiedlich verläuft, stellt keinen Widerspruch zur kantischen Theorie dar. Worauf es hier ankommt ist, dass die Zeit nicht willkürlich verläuft, sondern der Verlauf der Zeit in einem gesetzlichen Zusammenhang (der einen Erfahrung) steht. Rosenberg illustriert dies anhand eines anderen Beispiels, so verweist er auf zwei Träume, die zwar in sich einen kohärente zeitliche Struktur aufweisen, voneinander aber getrennt sind und in keinem zeitlichen Zusammenhang stehen. Damit wäre genau die Situation geschaffen, die durch die Beharrlichkeit der einen Substanz vermieden wird. Vgl. Rosenberg (2005), 209-210.

32 Vgl. KrV, A 110/B 284; siehe oben Anm. 23.

33 Wären die Voraussetzungen, unter denen Erfahrung gemacht werden kann, von Subjekt zu Subjekt unterschiedlich, so könnte auch nicht von den Bedingungen der Möglichkeit der Erfahrung auf eine allgemeine Gesetzmäßigkeit geschlossen werden.

34 Die Frage, ob Kant diese Auffassung direkt von Leibniz oder aus einem der einschlägigen Metaphysikhandbücher seiner Zeit übernommen hat, ist dabei nebensächlich. Nahezu alle deutschen Philosophen des 18. Jahrhunderts sind der Meinung, dass die Substanz ein einfaches Subjekt der Kraft ist.

35 Vgl. auch B 288, 300, A 401: „[...] ein Ding, als Subjekt an sich, ohne wiederum Prädikat von einem andern zu sein, [...].“; Refl 5873, AA 18: 373.24-28: „Wir können Dinge nur durch praedicate erkennen, also entstehen und vergehen nur als praedicate; nun kan man von etwas als praedicat keinen Begrif haben ohne subiect, also muß das Subiect bleiben bey allem, was vergeht; sonst können wir das Vergehen selbst nicht durch Erfahrung erkennen."

36 Vgl. hierzu auch Refl 5650, AA18: 298.13-16: „Substantz ist das letzte Subiect der Realitaet. Ihr Verhaltnis zum [dieser] Daseyn dieser heißt Kraft, und diese ist es allein, wodurch die Existentz der Substanz bezeichnet wird und worin ihre Existenz auch selbst besteht." 
das letzte Subjekt aller Veränderung ist die Substanz selbst keiner Veränderung unterworfen.

Mit der Definition der Substanz als einem Subjekt, von dem alles ausgesagt wird und das jeder Veränderung zugrunde liegt, während es selbst von nichts Anderem prädiziert werden kann, gibt Kant die Substanzdefinition des Aristoteles aus dessen Kategorienschrift wieder. ${ }^{37}$ Der Bezug zu Aristoteles wird von Kant selbst sowohl in der Kritik der reinen Vernunft als auch in den Prolegomena nahe gelegt. ${ }^{38}$ Neben der Tatsache, dass sich die Kategorien des Aristoteles fundamental von denen Kants unterscheiden, ergänzt Kant die Aristotelische Ausführung um wesentliche Punkte hinsichtlich des Verhältnisses von Kausalität und Substanz. Die Zusammenführung von physikalischen und logischen Bestimmungen im Substanzbegriff kann als ein Erbe angesehen werden, das Kant von Leibniz bzw. der Leibniz-Wolff'schen Schule übernommen hat, und muss als eine genuine Erweiterung des Aristotelischen Substanzbegriffs der Kategorienschrift begriffen werden. In dem hier besprochenen Zusammenhang ist besonders interessant, dass Aristoteles seine eigene, in der Kategorienschrift gelieferte Substanzdefinition in Metaphysik VII.3 kritisiert und revidiert. ${ }^{39}$ Dort führt Aristoteles aus, dass, wenn die Substanz immer nur Subjekt und niemals Prädikat sein kann, sie folglich, da sie sich nicht verändert, sondern mit sich identisch bleibt und das Identische nicht das immer wieder Andere sein kann, gegen alles Andere unbestimmt sein muss. So ist die Substanz als letztes Subjekt jeder Prädikation Materie, und zwar Materie im Sinne der Aristotelischen Hyle. ${ }^{40}$ Eine solche Materie kann als ein an sich Unbestimmtes keine reelle Unterscheidung zulassen, d.h. sie ist reine Möglichkeit. Wenn die Materie jedoch formlos ist und keine reelle Unterscheidung zulassen kann, ist sie zuletzt nur eine einzige. ${ }^{41}$ Aristoteles bemerkt sogleich, dass die Definition der Substanz als letztes Subjekt einer jeden möglichen Prädikation unzureichend ist, da wichtiger noch als die genannte Eigenschaft die Tatsache ist, dass

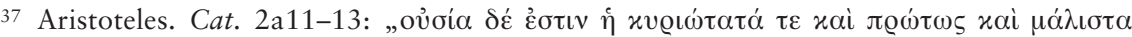

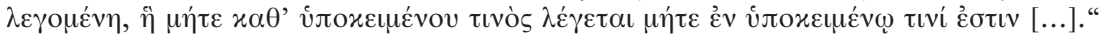
Sowie: Cat. 2b15-17; 2b37-3a1; 3a7-9; 3b10; 4a10-11.

38 Vgl. Prol $\$ 39$, AA 04: 323.10-16

$39 \mathrm{Zu}$ dem Verhältnis zwischen Kategorienschrift und Metaphysik siehe Rainer Thiel: Aristoteles' Kategorienschrift in ihrer antiken Kommentierung. Tübingen 2004.

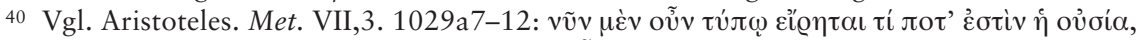

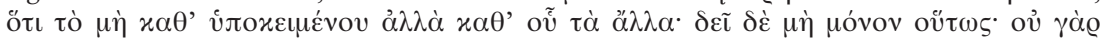

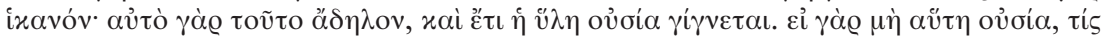

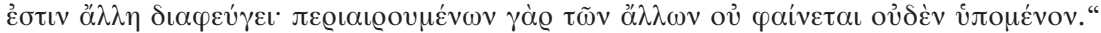

41 Die kantische Materie unterscheidet sich insofern von der aristotelischen, dass Kant diese als substantia phaenomenon notwendig im Raum sieht im Gegensatz zu Aristoteles. Vgl. Aristoteles, Met. 1029a16-21. Das ändert jedoch nichts an der Tatsache, dass die Substanz im Rahmen der kantischen Konzeption nur eine sein kann, da der Raum selbst auch nur einer ist, und jeder empirische Raum nur als Einschränkung des einen Raumes denkbar ist. Die kantische Materie weist darüber hinaus Ähnlichkeiten mit der stoischen Materiekonzeption auf, denn auch für die Stoiker ist die Materie nur eine, die jeder eigenschaftsmäßig bestimmten Substanz zugrunde liegt. Vgl. Long, A.A., Sedley, D. N.: Die hellenistischen Philosophen, Texte und Kommentare. Stuttgart, Weimar 2000, 28A, D, E. 
die Substanz ein $\tau o \delta \varepsilon \tau \iota$, d.h. ein dieses da, also etwas individuell Bestimmtes sei. ${ }^{42}$ Eine so verstandene Substanz wäre hingegen nur eine einzige, absolut unbestimmt und gerade kein konkreter Gegenstand. Die Charakterisierung der Substanz als ein Subjekt, welches niemals Prädikat ist, bleibt damit offensichtlich defizitär, wenn die Substanz nicht um andere Bestimmungen ergänzt wird.

Dies hat Leibniz dazu geführt, die Substanz als ein „être capable d'action“ zu bestimmen. ${ }^{43}$ Nur ist die Kraft, die Leibniz im Sinn hat, teleologisch gefasst und die Monaden überhaupt sind als letzte Einheiten der Wirklichkeit zugleich die Formprinzipien oder Entelechien der Materie. In logischer Hinsicht drückt sich bei ihm die Bestimmung der Substanz als letztes Subjekt einer jeden Prädikation in dem Gedanken aus, die Substanzen als notiones completae zu begreifen, sodass jedes mögliche Prädikat bereits in der Substanz enthalten ist und lediglich durch die Kraft der Monade, d.h. ihr perzeptionales Streben, realisiert wird. ${ }^{44}$ Beides fasst Leibniz unter die inneren Bestimmungen einer Substanz. Ohne diese inneren Bestimmungen kann es nach Leibniz offensichtlich kein principium individuationis für die Substanz geben und mithilfe der inneren Bestimmungen ist es Leibniz gelungen, die aristotelische Substanzdefinition in beiden Aspekten, nämlich das letzte Subjekt jeder Prädikation und zugleich ein individuell bestimmtes Dasein auszudrücken, zu erfüllen.

Was soll man sich aber unter der kantischen Substanz im Raum - der substantia phaenomenon - vorstellen, die selbst unveränderlich, unvergänglich, zu allen Zeiten mit sich identisch, beharrlich jeder möglichen Veränderung zugrunde liegt? Kant selbst äußert sich an mehreren Stellen in der KrV dazu. So ist für ihn nichts Beharrliches in der Anschauung denkbar, das wir mit dem Begriff der Substanz belegen können, als bloß die Materie. ${ }^{45}$ Mit einem Beispiel versucht Kant, seine Substanzkonzeption zu verdeutlichen:

Ein Philosoph wurde gefragt: wie viel wiegt der Rauch? Er antwortete: ziehe von dem Gewichte des verbrannten Holzes das Gewicht der übrigbleibenden Asche ab, so hast du das Gewicht des Rauchs. Er setzte also als unwidersprechlich voraus: daß selbst im Feuer die Materie (Substanz) nicht vergehe, sondern nur die Form derselben eine Abänderung erleide. (KrV, A 185/B 228)

Kant identifiziert die Substanz mit Materie, die auch dann erhalten bleibt, wenn die bestimmte Form, wie z. B. die des Holzes, aufgelöst wird. Aber ist die Materie selbst damit nicht bestimmungslos? Wenn ja, wie kann diese absolut formlose

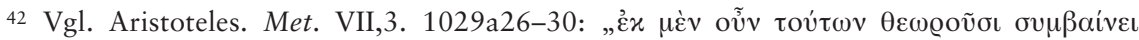

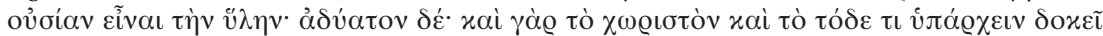

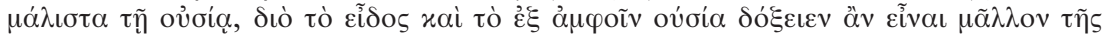

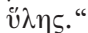

43 Vgl. Leibniz: Principes de la Nature et de la Grace, fondés en raison $\$ 1$, GP VI, 598. Zur Entwicklung des Leibnizschen Substanzbegriffs im Ausgang von Aristoteles siehe Woolhouse, Roger S.: Descartes, Spinoza, Leibniz, The concept of substance in seventeenthcentury metaphysics. London, New York 1993, 54-73.

44 Vgl. Leibniz: Eclairissement des difficultés que Monsieur Bayle a trouvée dans le syteme nouveau de l'union de l'ame et du corps, GP IV, 518.

45 Vgl. KrV, B 278: „[...] so haben wir sogar nichts Beharrliches, was wir dem Begriffe einer Substanz als Anschauung unterlegen könnten, als bloß die Materie [...].“ 
Materie vorstellig gemacht werden? Im transzendentalen Verstande darf die Materie keine Bestimmung haben, da wir a priori über Materie nichts aussagen können. ${ }^{46}$ So kann gefragt werden, ob die von Kant gelieferte Definition der Materie als dem absolut Beharrlichen im Raume ausreicht, um ein Quantum von unterscheidbaren Substanzen zu bestimmen. Wie kann eine absolut beharrliche Materie, ein Dasein zu aller Zeit, zugleich eine Menge voneinander unterscheidbarer Einzelsubstanzen sein, oder diese aus sich hervorbringen? Wollte man mit Thöle behaupten, die Erhaltung des Quantums der Substanz beträfe die Anzahl numerisch verschiedener Substanzen, so erweist sich die Beantwortung dieser Frage als die crux der gesamten Interpretation. ${ }^{47}$ Von Leibniz wissen wir, dass eine Substanz zur Individuation innerer Bestimmungen bedarf. Kant stimmt Leibniz grundsätzlich darin $\mathrm{zu}^{48}$ nur kann für ihn eine Substanz, die im Raume erscheint, keine inneren Bestimmungen haben. „Was ihr innerlich zukomme, suche ich in allen Teilen des Raumes, den sie einnimmt, und in allen Wirkungen, die sie ausübt, und die freilich nur immer Erscheinungen äußerer Sinne sein können. Ich habe also zwar nichts Schlechthin-, sondern lauter Komparativ-Innerliches, das selbst wiederum aus lauter äußeren Verhältnissen besteht. “ (KrV, A 277/B 333) Das schlechthin Innerliche dagegen ist in den Augen Kants eine bloße Grille, „denn diese ist überall kein Gegenstand für den reinen Verstand; das transscendentale Object aber, welches der Grund dieser Erscheinung sein mag, die wir Materie nennen, ist ein bloßes Etwas, wovon wir nicht einmal verstehen würden, was es sei, wenn es uns auch jemand sagen könnte. “ (KrV, A 277/B 333) Materie ist Erscheinung und an den Begriff des Raumes gebunden; somit dann auch an das erkennende Subjekt, da der Raum die subjektive Bedingung der Anschauung ist. Weil die Materie in dieser Hinsicht keine selbstständige Existenz hat, kann sie auch keine absolut für sich bestehende Substanz mit selbstständiger Existenz sein, welche als solche notwendig innere Bestimmungen haben müsste. Die Materie ist substantia phaeno-

46 In dieser Hinsicht muss die Materie der KrV scharf von derjenigen der MAN unterschieden werden. 1786 legt Kant der Materie das empirische Prädikat der Bewegung bei, das es ihm erlaubt, die Substanz der Materie mit dem Beweglichen im Raum zu identifizieren und als materielle Substanz zu individuieren. Für die KrV ist diese von einer ganzen Reihe von Interpreten vorgeschlagene Lösung ausgeschlossen. Vgl. Saville, Anthony: Kant's Critique of pure Reason - an Orientation to the central Theme. Malden, Oxford, Victoria 2005, 69-70; Thöle, usw. Die Substanz in der KrV also im Rückgriff auf die MAN erklären zu wollen, darf nicht geschehen, denn die MAN setzen die KrV, und zwar insbesondere die Analogien der Erfahrung voraus.

47 Vgl. Thöle (1998), 275-276.

48 Für die vorkritische Philosophie ist das klar, dass er es aber auch in der $\mathrm{KrV}$ noch so sieht, wird z.B. durch folgende Äußerungen Kants in der KrV dokumentiert: „Als Objekt des reinen Verstandes, muß jede Substanz dagegen innere Bestimmungen und Kräfte haben, die auf die innere Realität gehen.“ (KrV, A 265/B 321); „Die Substanzen überhaupt müssen etwas Inneres haben, was also von allen äußeren Verhältnissen, folglich auch der $\mathrm{Zu}$ sammensetzung, frei ist.“ (KrV, A 274/B 331); „Dergleichen transzendente Ideen haben einen bloß intelligibelen Gegenstand, welchen als ein transzendentales Objekt, von dem man übrigens nichts weiß, zuzulassen, allerdings erlaubt ist, wozu aber, um es als ein durch seine unterscheidende und innere Prädikate bestimmbares Ding zu denken, wir weder Gründe der Möglichkeit [...] noch die mindeste Rechtfertigung [...] auf unserer Seite haben [...].“ (KrV, A 565-566/B 593-594; Hervorhebung: Vf.) 
menon, d.h. eine Substanz, die im Raum erscheint, und hat als solche auch keine inneren Bestimmungen. ${ }^{49}$ Für die Substanz ergibt sich somit folgendes Problem: Dass die Materie nicht aus dem Einfachen zusammengesetzt sein kann, steht für den kritischen Kant fest und folgt aus mehreren Annahmen. Da das Einfache notwendig innere Bestimmungen braucht, muss das Ganze der Materie den Teilen vorausgehen. So gesehen wären die durch den Raum unterschiedenen Einzelsubstanzen im Verhältnis zu der einen primären Substanz oder Materie sekundär. Das Problem würde somit nicht gelöst werden, sondern reproduziert sich lediglich. So würde nicht an einzelnen Substanzen die Zeit vorstellbar gemacht werden, sondern die ihnen allen zugrunde liegende Materie würde diese Funktion übernehmen. Die sekundären Substanzen würden dann hinsichtlich der Vorstellbarkeit der einen Zeit und deshalb auch für die Einheit der Erfahrung keine Rolle spielen. Die Substanz als das Beharrliche im Dasein zu aller Zeit kann somit nur eine sein, die jede kategorial bestimmte materielle Substanz in sich enthält. Neben der aristotelischen Materie lässt sich auch - und das vor allem im Hinblick auf das Kantische Spätwerk - die Substanz, so wie sie uns im Raum erscheint als raumerfüllendes Kontinuum bewegender Kräfte deuten. Ohne den nachfolgenden Überlegungen zum Verhältnis der Entwürfe „Übergang 1-14“ des Nachlasswerkes und der ersten Analogie der Erfahrung vorgreifen zu wollen, kann bereits hier angemerkt werden, dass die Substanz, wie sie in der KrV vorgestellt, und die Materie überhaupt, wie sie in dem genannten Entwurfzusammenhang des OP behandelt wird, einerseits deutliche (funktionale) Übereinstimmungen, andererseits ebenso deutliche konzeptionelle Brüche erkennen lässt. Was die Übereinstimmungen anbelangt, zeigt sich, dass Kant auch in „Übergang 1-14“, wie im Folgenden dargetan werden wird, die Einheit der Zeit (und des Raumes) mit der Einheit der Substanz verbindet, und darüber hinaus mit der Unterscheidung einer primären Materie (Substanz) und einer Mannigfaltigkeit sekundärer Materie(n)/Substanzen operiert. Der grundlegende Unterschied dieser beiden Vorstellungen von Materie ist mit dem Begriff des Zusammenhanges verbunden. Die sogenannte Materie überhaupt stellt das unzusammenhängende Pendant zu jedweder Art von physischem Körper dar. Wenn hier davon die Rede ist, dass die Materie überhaupt unzusammenhängend ist, so ist damit nicht ausgedrückt, dass in ihr ein leerer Raum anzutreffen ist, da Kant die transzendentale Unmöglichkeit nicht bloß komparativ leerer Teile des Erfahrungsraumes in das Zentrum seines Existenzbeweises der Materie überhaupt stellt. Stattdessen ist damit lediglich das Fehlen eines körperlichen Zusammenhanges gemeint. Der Zusammenhang mikro- wie auch makrophysikalischer Körper wird durch die primären Bewegkräfte der Materie über-

49 Kant führt gegen Leibniz aus, dass der Raum selbst ein Unterscheidungskriterium liefert. Als Beispiel nennt er Wassertropfen, die zwar ihren inneren Bestimmungen nach, d.h. der Qualität und der Quantität nach gleich sind, sich dennoch unterscheiden, weil sie sich an verschiedenen Orten im Raum befinden. Die unterschiedlichen Raumstellen dienen somit als ein Individuationskriterium. Dem kann erwidert werden, dass die Verschiedenheit der Örter zwar ein notwendiges Kriterium der Individuation darstellt, aber nicht hinreichend ist. Denn auch an der einen Substanz im Raum können unterschiedliche Örter bestimmt sein. 
haupt konstituiert. Die sekundären Bewegkräfte der körperlichen Materie(n) sind als solche direkt wahrnehmbar, während die Bewegkräfte der ersten Materie einzig Gegenstand indirekter Erfahrung sein können, d.h. als transzendental notwendig gefolgert werden. ${ }^{50} \mathrm{Im}$ Bestreben, eine grundlegende Systematisierung aller möglichen bewegenden Kräfte der Materie als Basis einer im Vollsinne wissenschaftlichen, i.e. systematischen Naturwissenschaft vorzulegen, legt Kant der Materie überhaupt Bestimmungen bei, die das Substrat nicht mehr, im Sinne einer aristotelischen Hyle, als aller Bestimmung fähig erscheinen lässt. Materie überhaupt wird in „Übergang 1-14“ als (i) unwägbar, (ii) unsperrbar, d.h. durch keine andere Materie auf ein bestimmtes Volumen begrenzbar, (iii) unzusammenhängend, und (iv) unausleerbar, was ihr Quantum und ihre Bewegung anbelangt, charakterisiert. ${ }^{51}$ Mit diesen Eigenschaften, die Kant in „Übergang 1-14“ der Materie überhaupt zuspricht, erweist sie sich nicht mehr aller Bestimmungen fähig. Sie ist zwar „formlos"52, mithin ohne Gestalt und daher jeder formalen Bestimmung offen, nicht aber offen für jede Bestimmung überhaupt. Die Materie überhaupt in „Übergang 1-14“ kann zumindest in Bezug auf ihre Konzeption nicht mit der Vorstellung von Materie in der $\mathrm{KrV}$ und ebenso wenig mit der sogenannten Materie überhaupt in den MAN identifiziert werden. Es wird jedoch in der nachstehenden Darstellung des Verhältnisses von der ersten Analogie der Erfahrung und der „Übergang“-Entwürfe des OP zu zeigen sein, dass eine funktionale Vergleichung beider Konzepte einer Materie bzw. einer Substanz zur Gewährleistung der Zeit- und mithin Erfahrungseinheit durchaus möglich ist.

\section{Kants Erste Analogie der Erfahrung und der Entwurfzusammenhang „Übergang 1-14“ des kantischen Nachlasswerkes}

Die vorangegangene Betrachtung der Ersten Analogie der Erfahrung in beiden Ausgaben der KrV hat deutlich gemacht, dass es zur Gewährleistung einer (auf den Bereich der Erfahrung bezogen) absoluten Einheit der Zeit und mithin zur Einheit der Erfahrung selbst notwendig ist, von der einen Substanz als dem Substrat der Zeitvorstellung überhaupt auszugehen. Wenngleich im Text der Ersten Analogie auch von Substanz im Plural die Rede ist, und vor allem der unabhängig vom eigentlichen Beweistext betrachtete Grundsatz der Ausgabe A die Möglichkeit eröffnet, unter dem Gegenstand selbst sowohl (i) eine individuelle Substanz, d.h. einen durch ontologische Prädikate bestimmten Gegenstand überhaupt, wie auch (ii) das Eine und schlechthin Zugrundeliegende im Sinne eines Substantiale oder Hypokeimenon zu verstehen, kann dasjenige Substrat, welches die Zeiteinheit vorstellt, nur ein solches im Sinne der zweiten Alternative sein. Diesem einen unveränderlichen Substrat stünde das Wandelbare als alles irgend prädikativ Be-

50 Vgl. OP, AA 21: 536.17-537.02.

51 Vgl. die erste vollständige Aufzählung dieser Eigenschaften in „Anmerkung 2“ des Entwurfs „Übergang 4“ OP, AA 21: 231.28-232.12.

52 OP, AA 21: 215.15. 
stimmte gegenüber, was die gegenstandskonstituierende ontologische Prädikation durch Begriffe von einem Gegenstand überhaupt, die reinen Verstandesbegriffe einschließt. Bereits diese Feststellung rückt die Substanz der Ersten Analogie der Erfahrung in ihrer Funktion als Garant einer absolut einheitlichen Zeit in deutliche Nähe zu jenem Weltstoff, dessen Existenz Kant in den sogenannten Ätherbeweisen der Entwürfe „Übergang 1-14“ a priori zu deduzieren versucht.

Die Entwürfe „Übergang 1-14“, deren Entstehung in die Sommermonate des Jahres 1799 fällt, nehmen zwar bezogen auf das Nachlasswerk in seiner Gesamtheit nicht eben viel Raum ein; nach Meinung der Mehrzahl der Interpreten des OP stehen sie jedoch im Zentrum desselben. ${ }^{53}$ In „Übergang 1-14“ zeigen sich die deutlichsten Brüche mit der Systemkonzeption der KrV, und zwar ungeachtet aller sonst zu konstatierenden Kontinuität im Denken Kants, die sich auch anhand des $O P$ belegen lässt.

Kant stellt in „Übergang 1-14“ im Zuge einer verglichen mit 1786 und 1790 neuartigen Systematisierung der Naturwissenschaft der/-n körperlich zusammenhängenden Materie(n) direkt empirisch wahrnehmbarer Einzeldinge etwas gegenüber, das er als die „Materie überhaupt (formlos)“ (OP, AA 21: 215.14-15) bezeichnet. Als Pendant der physischen Körper (womit nicht allein makro-, sondern ebenso mikrophysikalische Gegenstände gemeint sind) kommen der Materie überhaupt bestimmte Eigenschaften zu (s.o.), die ihrerseits Negationen der Eigenschaften ihres Pendants, der (körperlich) zusammenhängenden Materie sind. Nicht zuletzt aufgrund dieser Neukonzeption, innerhalb welcher auch der Begriff einer Materie überhaupt eine andere Bedeutung als in 1786 erhält, werden Zirkelprobleme in der Körperbildungstheorie der MAN ausgeräumt. ${ }^{54}$ Kant reagiert jedoch mit dieser neuen Systematisierung der Naturwissenschaft nicht allein auf Probleme seiner Materietheorie. Die naturphilosophischen Aspekte werden in „Übergang 1-14“ vielmehr in einen transzendentalphilosophischen Rahmen integriert, innerhalb dessen der Materie überhaupt eine erfahrungskonstitutive Funk-

53 Vgl. e.g. Förster, für den zwar die Selbstsetzungslehre der letzten Konvolute als „clearly the culmination of Kann's last work " zu betrachten ist, Förster, Eckart: Kant's Final Synthesis. An Essay on the Opus postumum. Cambridge, London 2000, 75, der jedoch gleichermaßen feststellt, dass „the drafts „Übergang 1-14“ with their ether proofs [...] occupy a central position in the Opus postumum “ (ebd. 19); diese Auffassung bestätigt auch Tuschling, dem zufolge „Übergang 1-14“ „occupies the central period among the papers of the Opus postumum“, vgl. Tuschling, Burkhard: „Apperception and Ether: On the Idea of a Transcendental Deduction of Matter in Kant's Opus postumum “, in: Kant's Transcendental Deductions. The Three ,Critiques' and the ,Opus postumum'. Ed. by E. Förster. Stanford 1989, 201. Mathieu spricht bezogen auf die Versuche Kants, in „Übergang 1-14“ einen a priori Beweis der Existenz des Äthers vorzulegen, von dem „erstaunlichsten Teil des OP“, vgl. Mathieu, Vittorio: Kants Opus postumum. Hrsg. von Gerd Held. Frankfurt a. M. 1989, 111; Schulze zufolge sind „die Ätherbeweise nicht nur argumentativer Höhepunkt des Nachlasswerks, sondern auch Ausgangspunkt von dessen weiterer Entwicklung“, vgl. Schulze, Stefan: Kants Verteidigung der Metaphysik. Eine Untersuchung zur Problemgeschichte des Opus Postumum. Marburg 1994, 30.

54 Zum Zirkelproblem in der Materietheorie der MAN vgl. Emundts, Dina: Kants Übergangskonzeption im Opus postumum. Zur Rolle des Nachlaßwerkes für die Grundlegung der empirischen Physik. Berlin, New York 2004, 74-117. 
tion zukommt. Erfahrung im Sinne Kants ist eine absolut einheitliche Erfahrung. Dies hatte die erste Fassung der transzendentalen Deduktion der Kategorien deutlich herausgestellt. ${ }^{55}$ Ebenso wie es nach der transzendentalen Ästhetik der KrV nur einen Raum und eine Zeit als unendliche gegebene Größen gibt, und Raum und Zeit in plurali einzig als Limitationen dieser zugrunde liegenden Einheit begriffen werden können, verhält es sich mit der Einheit der Erfahrung. Wahrnehmungen als direkte Erfahrungen, deren distributive Einheit dem reinen Verstand des transzendentalen Subjekts entspringt, verweisen auf das selbst nicht wahrnehmbare Ganze der Erfahrung. Dieses Ganze in materieller Absicht ist der eine Weltstoff als Inbegriff dessen, was zur Möglichkeit der Erfahrung gegeben sein kann: die eine Materie überhaupt als ein raumerfüllendes Kontinuum aller bewegenden Kräfte der Materie. Bereits die ersten Entwürfe aus dem Zusammenhang von „Übergang 1-14“ zeigen das wechselseitige Bedingungsverhältnis der Konstituentien von Erfahrung als einer absoluten Einheit. Wenn Kant in „Übergang 2“ feststellt, dass im Ganzen „nur Ein Raum und nur Eine Zeit und nur Eine Materie“ (OP, AA 21: 224.03-04) existieren, so wird dasjenige, was als die eine Materie überhaupt diese Konstituentien einer sinnlich apprehendierbaren raumzeitlichen Welt zu wirklichen Gegenständen der Erfahrung macht, zu dem „Princip der Moglichkeit der Einheit des Ganzen möglicher Erfahrung“ (OP, AA 21: 224.12-13). Der Weltstoff der Entwürfe „Übergang 1-14“ ist nun genau dieses materiale Substrat, das die Einheit der Zeit vorstellig macht, von welcher wiederum die Einheit der Erfahrung in gleichem Maße abhängt, wie von der Einheit des Erfahrungsraumes. Insofern ist es keineswegs überzogen, den Weltstoff der Entwürfe „Übergang 1-14“ mit der Substanz der ersten Analogie der Erfahrung zu identifizieren. Wenngleich der Grundsatz der ersten Analogie isoliert betrachtet noch die Frage offengelassen hat, ob es sich bei dem Beharrlichen um eine individuelle Substanz unter vielen Substanzen oder aber um die eine und einzige Substanz als das letzte Substrat der Zeiteinheit und in logischer Absicht jeder möglichen Prädikation handelt, hat bereits die Betrachtung von Grundsatz und Beweis in 1781 diese Pluralität möglicher Deutungen negiert. ${ }^{56}$

Dies wird im Text der ersten Analogie in 1787 noch weitaus deutlicher herausgestellt. Hier ist bereits innerhalb des isoliert betrachteten Grundsatzes der ersten Analogie die Möglichkeit ausgeschlossen, unter dem beschriebenen Beharrlichen eine individuelle Substanz zu verstehen. Vielmehr begegnet die Substanz in kollektiver Einheit als Möglichkeitsbedingung der einen Erfahrung. Nicht erst in den

55 Vgl. nochmals KrV, A 110.

56 So schreibt Kant bereits im Kontext seines (ersten) Beweises zum Grundsatz der Beharrlichkeit der Substanz, „dieses Beharrliche [i.e. das grundlegende kontinuierliche materiale Substrat, Vf.] an den Erscheinungen“ sei „das Substratum aller Zeitbestimmung, folglich auch die Bedingung der Möglichkeit aller synthetischen Einheit der Wahrnehmungen, d. i. der Erfahrung“ (KrV, A 183). Nicht als bloß relatives Substrat einer kontextvarianten relativen Zeitvorstellung, sondern als das „Substratum der empirischen Vorstellung der Zeit selbst, an welchem alle Zeitbestimmung allein möglich ist" (ebd.). Nicht also irgend eine Substanz, sonder die eine und einzige Substanz als Korellatum der Vorstellung einer einheitlichen Zeit und mithin als Möglichkeitsbedingung der einen und einzigen allbefassenden Erfahrung. 
„Übergang“-Entwürfen des OP, sondern bereits innerhalb der ersten Analogie der Erfahrung in der KrV findet die von Kant in seinem Kapitel „Von dem Transzendentalen Ideal“ als unzulässig verworfene dialektische Verwandlung der „distributive[n] Einheit des Erfahrungsgebrauchs des Verstandes in die kollektive Einheit eines Erfahrungsganzen“ (KrV, B 610) statt! Der Weltstoff in seiner Auffassung als die eine Substanz in der Erscheinung macht, wie Kant in „Übergang 14“ deutlich macht, ein „absolutes, für sich bestehendes Gantze“ (OP, AA 22: 610.04) aus. Seine Inexhaustibilität sub specie totalitatis bezieht sich sowohl auf die Bewegungskraft, wie auch auf das Gesamtquantum des einen Weltstoffs, der als solcher das „All der Materie“ (OP, AA 21: 210.01 und 592.13) darstellt. Insofern liefert der Weltstoff den in der ersten Analogie geforderten absolut beharrlichen Parameter der Zeitbestimmung. Er ist das quantitativ konstante, absolut beharrliche Substrat einer Erfahrung, deren raumzeitliche Einheit material begründet ist. Dennoch ist im Zusammenhang der ersten Analogie der Erfahrung an zwei Stellen von Substanzen die Rede. ${ }^{57}$ Kant stellt damit die radikal zu denkende Singularität der Grundlage der Zeitvorstellung nicht in Frage. Vielmehr eröffnet Kant allem Anschein nach die Differenz von bloß relativer Beharrlichkeit und der notwendig absoluten Beharrlichkeit der einen Substanz selbst. In der Wahrnehmung gibt es bestimmte Erscheinungen, die sich, im Sinne des reinen Verstandesbegriffs von Substanz, anderen gegenüber als subsistierend erweisen. Sie sind Substanzen relativ zu den ihnen inhärierenden akzidentellen Erscheinungen; nicht aber in einem absoluten Sinne. Ebenso sind sie in der (direkten) Erscheinung Substrate verschiedener relativer Zeitbestimmungen, indem sie sich als relativ beharrlich ihren Akzidenzen gegenüber erweisen. Für die Erfahrung überhaupt und ihre Einheit, die Zeit überhaupt oder auch den Raum als solchen bedarf es auf der Ebene des transzendentalen Subjekts der Substanz überhaupt, die der Vorstellung dieser absoluten Einheit korreliert.

Abschließend lässt sich feststellen, das Kant im Entwurfzusammenhang „Übergang 1-14“ den transzendentalen Beweis einer notwendigen Möglichkeitsbedingung zu liefern versucht, die er jedoch bereits im Kontext der KrV, bspw. in der hier untersuchten Ersten Analogie der Erfahrung, wie auch in der Dritten Analogie implizit voraussetzt: die eine substantia phaenomenon, d.h. die Substanz, so wie sie im Raum erscheint, und den Inbegriff des zur Möglichkeit der Erfahrung als einer Einheit gegebenen Materials. Ungeachtet ihrer transzendentalen Notwendigkeit stellt diese Totalität der Materie für Kant in 1781 und 1787 noch ein „Problem ohne alle Auflösung “ dar. ${ }^{58}$

57 Vgl. nochmals KrV, A 188/B 231: „Substanzen (in der Erscheinung) sind die Substrate aller Zeitbestimmungen“, wie auch A 184/B 227: „diese Substanz oder Substanzen“.

58 Vgl. e.g. KrV, B 384: „das absolute Ganze aller Erscheinungen [die eine Natur/Erfahrung als Totum, Vf.] ist nur eine Idee, denn da wir dergleichen niemals im Bilde entwerfen können, so bleibt es ein Problem ohne alle Auflösung.“ 


\section{Ergebnis}

Ein Problem ohne Auflösung, an das bereits Heidegger in dem zu Beginn zitierten Zusammenhang erinnert, besteht jedoch mit der Mehrdeutigkeit des Begriffs von Substanz im Zusammenhang des kantischen Systems fort, für die auch das OP, zumindest was den betrachteten Entwurfzusammenhang anbelangt, keine neue Lösung anbietet. Wie gezeigt, erweist sich die eine Substanz als notwendig zur Einheit der Erfahrung. Zugleich bestimmt die Substanz aber auch den Gegenstand überhaupt, d.h. den Gegenstand der einen, objektiven Erfahrung. Diese Art des Gegenstandes nennt Heidegger Naturding, was nichts anderes meint als den Gegenstand einer Wissenschaft der Natur. Problematisch und ebenfalls ungelöst ist die nicht allein von Heidegger aufgeworfene Frage nach dem Bestimmungsgrund der Dinge in der Weise ihres alltäglichen Begegnens, oder, anders gewendet, die Frage nach dem Verhältnis von Gegenständen überhaupt und diesen konkreten Gegenständen der Erscheinung. Der reine Verstand stellt mit der Kategorie der Substanz das principium individuationis von Gegenständen überhaupt bereit. Dennoch muss, wenn alle darüber hinausgehenden Bestimmtheiten der Dinge als gegeben vorausgesetzt sein müssen, für diese ein Bestimmungsgrund angenommen werden, der nicht im reinen Verstand des transzendentalen Subjekts bestehen kann. Sind die einzelnen Dinge bereits in irgendeiner Weise da, bevor transzendentale Subjektivität sie überhaupt erst zu einzelnen Dingen macht?59 Oder anders gefragt, machen wir die Gegenständlichkeit der Dinge oder sind die Dinge in ihrer Gegenständlichkeit bereits durch die Sinnlichkeit gegeben? Wir haben gesehen, dass die Kategorie der Substanz für die Bestimmung der Gegenständlichkeit der alläglichen Dinge, d.h. der nur relativ beharrlichen Dinge unzureichend ist, da dadurch nur, wie Heidegger sagt, die Dinge als Gegenstände der Wissenschaft bestimmt sein können. Gibt es also darüber hinaus noch eine zweite Anwendungsart der Kategorie der Substanz? Kann die Kategorie der Substanz auch unschematisiert dasjenige zur Gegenständlichkeit bestimmen, was gar keine Substanz im Sinne Kants sein kann? Schon Hegel hat in seinen Schriften der Jenaer Zeit auf dieses zentrale Problem der kantischen theoretischen Philosophie hingewiesen. In der „Differenz ... “-Schrift macht er deutlich, dass „außer den objektiven Bestimmungen durch die Kategorien ein ungeheures empirisches Reich der Sinnlichkeit und Wahrnehmung, eine absolute Aposteriorität [bleibt], für welche keine Apriorität als nur eine subjektive Maxime der reflektierenden Urteilskraft aufgezeigt ist; d.h. die Nichtidentität wird zum absoluten Grundsatz erhoben. "60 Schärfer noch akzentuiert Hegel das Problem in „Glauben und Wissen“. Dort heißt es:

Eine solche formale Identität hat unmittelbar eine unendliche Nichtidentität gegen oder neben sich, mit der sie auf eine unbegreifliche Weise koaleszieren muß; so kommt denn auf eine Seite das Ich mit seiner produktiven Einbildungskraft oder vielmehr mit seiner synthetischen Einheit, die, so isoliert gesetzt, formale Einheit des Mannigfaltigen ist, neben dieselbe aber eine Unendlichkeit der Empfindungen und, wenn man will, der Dinge an sich, - welches

59 Vgl. zu diesem Problem auch Schmitt, Arbogast: Die Moderne und Platon. Stuttgart 2003, $178 \mathrm{ff}$.

60 Hegel, G. W. F.: Gesammelte Werke. Bd. 4. Hamburg 1968, 6. 
Reich, insofern es von den Kategorien verlassen ist, nichts anderes als ein formloser Klumpen sein kann, obschon es auch nach der Kritik der Urteilskraft als ein Reich der schönen Natur Bestimmtheiten in sich enthält, für welche die Urteilskraft nicht subsumierend, sondern nur reflektierend sein kann. Aber weil doch Objektivität und Halt überhaupt nur von den Kategorien herkommt, dies Reich aber ohne Kategorien und doch für sich und für die Reflexion ist, so kann man sich dasselbe nicht anders vorstellen als wie den ehernen König im Märchen, den ein menschliches Selbstbewußtsein mit den Adern der Objektivität durchzieht, daß er als aufgerichtete Gestalt steht, welche Adern der formale transzendentale Idealismus ihr ausleckt, so daß sie zusammensinkt und ein Mittelding zwischen Form und Klumpen ist, widerwärtig anzusehen, - und für die Erkenntnis der Natur und obne die von dem Selbstbewußtsein ihr eingespritzten Adern bleibt nichts als die Empfindung. ${ }^{61}$

Würde Kant im Nachlasswerk tatsächlich, wie manche Interpreten vermuteten, seinen formalen Idealismus in Richtung eines absoluten oder wahren Idealismus im Sinne Hegels modifizieren, wovon ungeachtet aller Brüche mit der Systemkonzeption der $\mathrm{KrV}$ in keiner Weise auszugehen ist, wäre eine Lösung dieser Schwierigkeit möglich. Auch wenn Kants Transzendentaler Idealismus im Nachlasswerk starke Modifikationen erfährt und Materie überhaupt zu den Möglichkeitsbedingungen der Erfahrung hinzukommt, liefern diese dennoch keinen Bestimmungsgrund der, im Sinne Hegels, absoluten Aposteriorität. Die eine Materie überhaupt der Entwürfe „Übergang 1-14“ begründet als funktionales Analogon der einen Substanz im Raum, wie sie die Erste Analogie vorgestellt hat, die Zeiteinheit und mithin die Einheit der Erfahrung. Das Problem der vielen einzelnen Substanzen in der Erscheinung in ihrer doppelten Auffassung als einerseits durch den reinen Verstand bestimmt, andererseits durch diesen unbestimmte und doch von irgend woher als bestimmt vorauszusetzende Dinge, wird von der Substanzspekulation Kants im Nachlasswerk genauso wie in der KrV unberührt gelassen, womit die Frage nach der Möglichkeit der Gegenständlichkeit der Dinge in ihrem alltäglichen Begegnen ungelöst bleibt, ja nicht einmal angesprochen wird, wie Heidegger - zumindest in diesem Punkt - treffend bemerkt.

61 Hegel (1968), 332. 